GSA Data Repository 2016347

\title{
Paleo-Asian Oceanic slab under the North China Craton revealed by carbonatites derived from subducted limestones
}

Chunfei Chen ${ }^{1}$, Yongsheng Liu ${ }^{1 *}$, Stephen F. Foley ${ }^{2}$, Mihai N. Ducea ${ }^{3,4}$, Detao $\mathrm{He}^{1}$, Zhaochu $\mathrm{Hu}^{1}$, Wei Chen ${ }^{1}$, Keqing Zong ${ }^{1}$

${ }^{1}$ State Key Laboratory of Geological Processes and Mineral Resources, School of Earth Sciences, China University of Geosciences, Wuhan 430074, China

${ }^{2}$ ARC Centre of Excellence for Core to Crust Fluid Systems, Dept. of Earth and Planetary Sciences, Macquarie University, North Ryde, New South Wales 2109, Australia.

${ }^{3}$ Department of Geosciences, University of Arizona, Tucson, AZ 85721, USA

${ }^{4}$ Faculty of Geology and Geophysics, University of Bucharest, Bucharest, Romania

\section{Analytical methods}

Whole rock samples were crushed in a corundum jaw crusher, and the resulting chips were handpicked to exclude the olivine, pyroxene and spinel xenocrysts. About $100 \mathrm{~g}$ of the sample was then powdered in an agate ring mill to less than 200 mesh.

The sample powders were digested by $\mathrm{HF}+\mathrm{HNO}_{3}$ in Teflon bombs and analyzed using an Agilent 7500a ICP-MS at the State Key Laboratory of Geological Processes and Mineral Resources, China University of Geosciences, Wuhan (SKL GPMR-CUG). The detailed sample digestion procedure for ICP-MS analysis is given in Liu et al. (2008a). Analyses of rock standards (BCR-2, BHVO-2 and AGV-2) indicate accuracies are better than $5 \%$ for major elements and $10 \%$ for trace elements.

The selected samples were leached using $1 \mathrm{~N}$ acetic acid to dissolve carbonate for $\mathrm{Sr}$ and $\mathrm{Nd}$ isotopic analyses. The leachate is believed to be the bulk carbonate component in the carbonatite. The chemical separation for $\mathrm{Sr}$ and $\mathrm{Nd}$ isotopic analyses is the same as the description by Gao et al. (2004). Sr-Nd isotopic ratios were determined using a Triton Ti TIMS at the SKL GPMR-CUG. The measured ${ }^{143} \mathrm{Nd} /{ }^{144} \mathrm{Nd}$ and ${ }^{87} \mathrm{Sr} /{ }^{86} \mathrm{Sr}$ ratios were normalized to ${ }^{146} \mathrm{Nd} /{ }^{144} \mathrm{Nd}=0.7219$ and ${ }^{88} \mathrm{Sr} /{ }^{86} \mathrm{Sr}=8.375209$, respectively. BCR-2 and GBW04411 were used as the monitor standard materials in this work. Our analytical results $\left({ }^{143} \mathrm{Nd} /{ }^{144} \mathrm{Nd}=0.512629 \pm 0.000006(2 \sigma)\right.$ for BCR-2 and ${ }^{87} \mathrm{Sr} /{ }^{86} \mathrm{Sr}=0.760033 \pm 0.000016(2 \sigma)$ for GBW04411) fit the recommend values $\left({ }^{143} \mathrm{Nd} /{ }^{144} \mathrm{Nd}\right.$ $=0.512636 \pm 0.000002(2 \mathrm{SD})$ for BCR-2; ${ }^{87} \mathrm{Sr} /{ }^{86} \mathrm{Sr}=0.76 \pm 0.000006$ (2SD) for GBW04411) within 
analytical uncertainty.

Major and trace elements of silicate minerals, carbonate matrix and phenocrysts were analyzed by LA-ICP-MS with a spot size of $44 \mu \mathrm{m}$ at the SKL GPMR-CUG. Element contents were calibrated against multiple reference materials without applying internal standardization. Details of operating conditions for LA-ICP-MS and data reduction strategy are same as Liu et al. (2008b) for silicate minerals and Chen et al. (2011) for carbonate. Off-line selection and integration of the background and the analyzed signals, along with time-drift correction and quantitative calibration, were performed by ICPMSDataCal (Liu et al., 2008b).

The polished sections were prepared for identifying moissanite and highly disordered graphite using a Thermo Scientific DRX dispersive Raman micro-spectrometer. In order to avoid possible contamination, $\mathrm{Al}_{2} \mathrm{O}_{3}$ abrasive papers only were used to prepare the polished sections. The detailed sample preparation procedure is given in Liu et al. (2015). 


\section{Data sources for carbonatite and limestone}

Carbonatites are compiled from a worldwide database (Bühn, 2008; Bell et al., 1982; Bell and Simonetti, 2010; Halama et al., 2008; Hoernle et al., 2002; Hou et al., 2015; Huang et al., 1995; Mitchell, 2005; Mourao et al., 2010).

Major elements in limestone were collected from worldwide (Armstrong-Altrin et al., 2003; Bellanca et al., 1997; Jin et al., 2009; Klein and Beukes, 1989; Tanaka et al., 2003; Tsikos et al., 2001). Trace elements in limestone collected from worldwide (Bellanca et al., 1997; Jin et al., 2009; Tanaka et al., 2003; Tsikos et al., 2001) are shown for comparison. 


\section{Detailed explanation, data sources and modelling parameters for Fig. 3}

The $\mathrm{CaO}$ versus Ni plot in Fig.3 (A) shows that the carbonates in the carbonatite intrusion trend to high Ni contents, in contrast to limestones. The solid curve is a simple mixing line between limestone and the Hannuoba peridotite (Rudnick et al., 2004): ticks on curves show $10 \%$ increments. Average of peridotites from Hannuoba (Rudnick et al., 2004) is shown for comparison. (B) Sr-Nd isotopic compositions of the Hannuoba intrusion (this paper). Aragonite veinlets (this paper), limestone (Keto and Jacobsen, 1988; Veizer et al., 1999), typical carbonatites (Bühn, 2008; Bell et al., 1982; Bell and Simonetti, 2010; Halama et al., 2008; Hoernle et al., 2002; Hou et al., 2015; Huang et al., 1995; Mitchell, 2005; Mourao et al., 2010), the Mesozoic carbonatites from Zhuolu (ZL) and Huairen (HR) (Yan et al., 2007), peridotite and garnet pyroxenite xenoliths (Rudnick et al., 2004; Xu, 2002), and alkali basalt (Song et al., 1990) from Hannuoba are shown for comparison. Average $\mathrm{Sr}$ and $\mathrm{Nd}$ contents $(\mathrm{Sr}=380 \mathrm{ppm} ; \mathrm{Nd}=0.66 \mathrm{ppm})$ in Silurian, Devonian and Carboniferous limestones from the Inner Mongolia derived from the Paleo-Asian Ocean (Liu, et al. unpublished data) were used in model calculations. Detailed parameters are listed in the table below.

The trend to high Ni contents shown by the carbonates could reflect mixing in of a lower proportion of peridotite $(\sim 10 \%)$ (Fig. 3A) than that of the trend to high ${ }^{143} \mathrm{Nd} /{ }^{144} \mathrm{Nd}(\sim 30 \%)$ (Fig. 3B). This could be attributed to the transfer of $\mathrm{Ni}$ from carbonatite melt to newly growth silicate minerals (i.e. clinopyroxene) during carbonatite-peridotite interaction.

\begin{tabular}{lcc}
\hline Compositional parameters & $\mathrm{Sr}$ & $\mathrm{Nd}$ \\
Limestone (ppm) & 380 & 0.66 \\
Limestone isotope ratios & $0.707-0.709$ & $0.5119-0.5123$ \\
Average of Hannuoba peridotites (ppm) & 14.6 & 1.09 \\
Average of Hannuoba peridotites isotopic ratios & 0.7038 & 0.51310 \\
\hline
\end{tabular}




\section{Figure DR1}
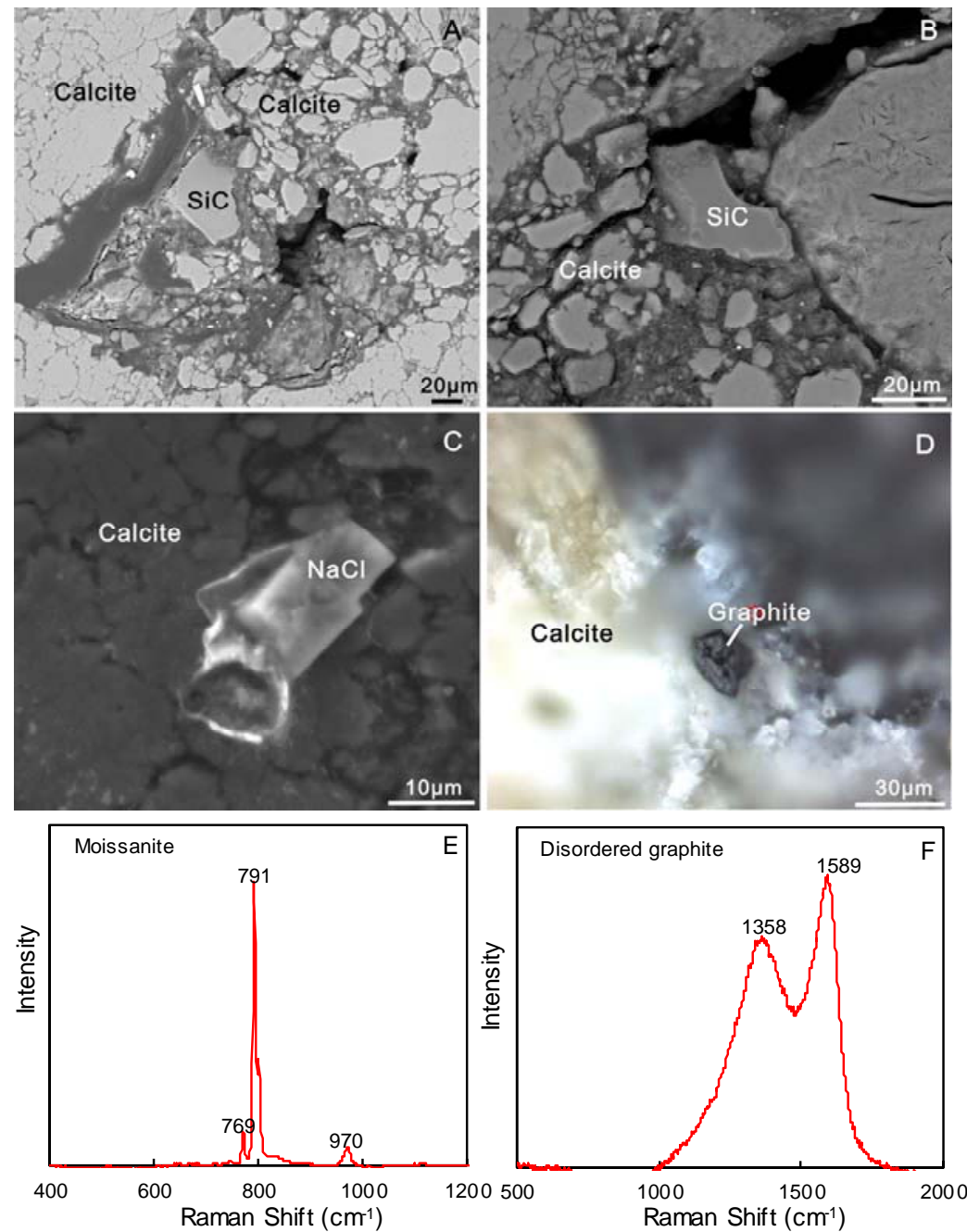

Moissanite (A and B), Na chloride (C) and graphite (D) coexisting with carbonate in the carbonatite intrusion. Raman shift of moissanite (E) and highly disordered graphite (F) in the carbonatite intrusion. 


\section{Figure DR2}

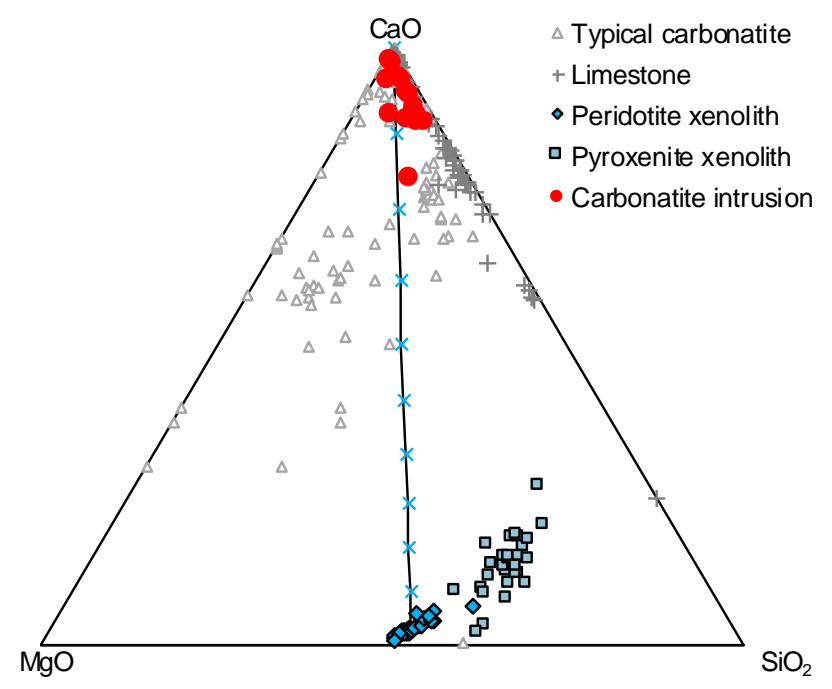

$\mathrm{CaO}-\mathrm{MgO}-\mathrm{SiO}_{2}$ diagram of the Hannuoba carbonatite intrusion (red circles). The solid line with crosses is a mixing line showing $10 \%$ increments between pure limestone and peridotite. Peridotite xenoliths (Rudnick et al., 2004) and pyroxenite veins (Liu et al., 2005; Xu, 2002) in peridotite xenoliths from Hannuoba, limestone (Armstrong-Altrin et al., 2003; Bellanca et al., 1997; Jin et al., 2009; Klein and Beukes, 1989; Tanaka et al., 2003; Tsikos et al., 2001) and typical carbonatites (Bühn, 2008; Bell et al., 1982; Bell and Simonetti, 2010; Halama et al., 2008; Hoernle et al., 2002; Hou et al., 2015; Huang et al., 1995; Mitchell, 2005; Mourao et al., 2010) are shown for comparison. 


\section{Figure DR3}

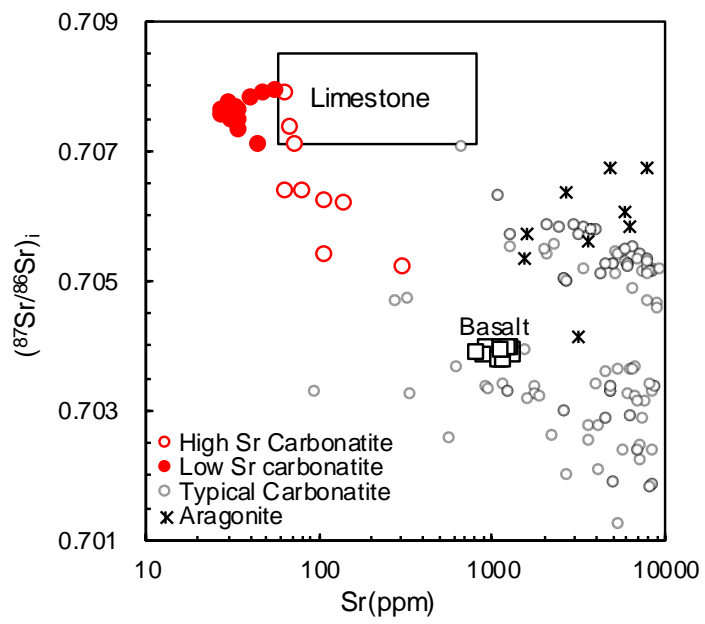

Variations of $\left({ }^{87} \mathrm{Sr}{ }^{86} \mathrm{Sr}\right)_{\mathrm{I}}$ with $\mathrm{Sr}$ contents for carbonates and aragonite veins in the Hannuoba carbonatite intrusion. The carbonatite intrusion can be classified into two types: low $\mathrm{Sr}$ carbonatite ( $\mathrm{Sr} 30 \sim 50 \mathrm{ppm}$ ) and high $\mathrm{Sr}$ carbonatite $(\mathrm{Sr}>60 \mathrm{ppm})$. The isotopic compositions are calculated back to $22 \mathrm{Ma}$ (age of the Hannuoba basalt). The typical carbonatites (data sources as in Figure DR2) and alkali basalts from Hannuoba are shown for comparison. Sr isotopic data for limestone are from Veizer et al. (1999). 


\section{Figure DR4}
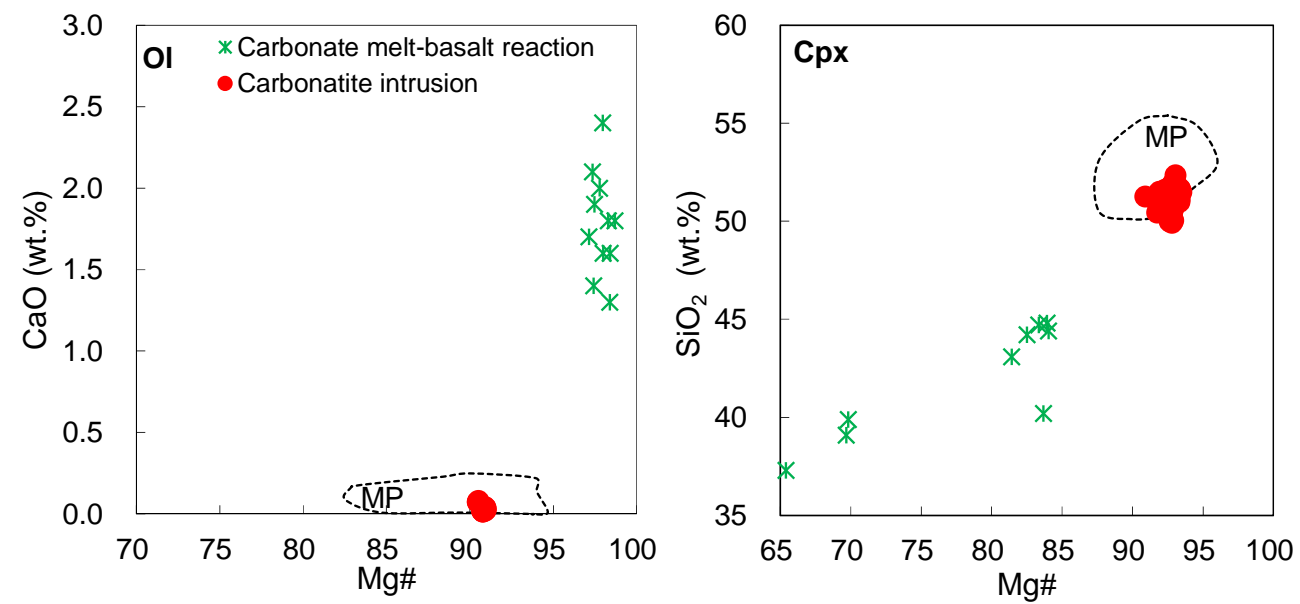

Plots of $\mathrm{Mg} \#$ versus $\mathrm{CaO}$ and $\mathrm{SiO}_{2}$ for $\mathrm{Ol}$ and $\mathrm{Cpx}$ xenocrysts from the Hannuoba carbonatite intrusion, eliminating the possibility that the carbonatite composition could be due to interaction between carbonate and basaltic melt. Data for $\mathrm{Ol}$ and $\mathrm{Cpx}$ formed by carbonate melt-basalt interaction are from Jolis et al. (2013). MP = Range of mantle peridotites from the North China Craton (e.g., Rudnick et al., 2004; Xu et al., 2013). 


\section{Figure DR5}

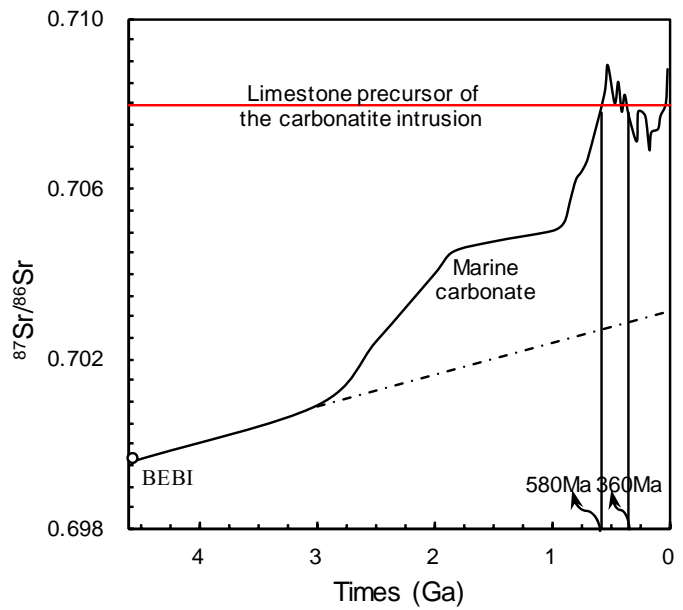

$\left({ }^{87} \mathrm{Sr} /{ }^{86} \mathrm{Sr}\right)_{\mathrm{I}}$ comparison between the limestone precursor of the Hannuoba carbonatite and the evolution line of marine carbonates (Veizer, 1985; Veizer et al., 1999). The intersection points infer that the limestone precursor of the carbonatite intrusion was certainly not older than $580 \mathrm{Ma}$, and most probably between 360 and $580 \mathrm{Ma}$. BEBI = Bulk-Earth best initial. 


\section{References not cited in main paper}

The temperatures of silicate macrocrysts were estimated using thermometer of $\mathrm{Cpx}+\mathrm{Opx}$ pairs (Wells, 1977).

(Burke et al., 2003) 


\section{Table DR 1}

Compositions of silicate mineral xenocrysts in the Hannuoba carbonatite intrusion Units are wt.\% for major elements and ppm for $\mathrm{Ni}$.

\begin{tabular}{|c|c|c|c|c|c|c|c|c|c|c|c|c|c|c|}
\hline \multirow[t]{2}{*}{ Num. } & \multicolumn{2}{|c|}{ JSB011 } & \multicolumn{3}{|c|}{ JSB029 } & \multicolumn{2}{|c|}{ JSB012 } & \multicolumn{3}{|c|}{ JSB024 } & \multicolumn{2}{|c|}{ JSB044 } & \multicolumn{2}{|c|}{ JSB054 } \\
\hline & $\mathrm{Cpx}$ & Opx & Cpx & $\mathrm{Ol}$ & Opx & Cpx & Opx & $\mathrm{Cpx}$ & Opx & $\mathrm{Ol}$ & Cpx & Opx & $\mathrm{Cpx}$ & Opx \\
\hline $\mathrm{SiO}_{2}$ & 51.2 & 54.8 & 50.8 & 39.0 & 54. & 50.1 & 55.4 & 51.3 & 55.4 & 39.5 & 50.6 & 54.3 & 51.1 & 55.7 \\
\hline $\mathrm{TiO}_{2}$ & 0.30 & 0.08 & 0.11 & 0.00 & 0.04 & 0.33 & 0.07 & 0.29 & 0.08 & 0.00 & 0.32 & 0.08 & 0.34 & 0.08 \\
\hline $\mathrm{Al}_{2} \mathrm{O}_{3}$ & 5.18 & 3.79 & 4.40 & 0.03 & 4.04 & 5.88 & 3.46 & 5.00 & 3.35 & 0.01 & 5.75 & 4.15 & 4.69 & 3.47 \\
\hline $\mathrm{TFeO}$ & 2.36 & 5.27 & 2.68 & 9.15 & 5.97 & 2.21 & 6.11 & 2.07 & 5.70 & 9.12 & 2.25 & 5.59 & 2.70 & 5.76 \\
\hline $\mathrm{MnO}$ & 0.08 & 0.13 & 0.09 & 0.14 & 0.14 & 0.07 & 0.14 & 0.08 & 0.14 & 0.13 & 0.08 & 0.13 & 0.08 & 0.14 \\
\hline $\mathrm{MgO}$ & 16.7 & 34.6 & 17.7 & 51.2 & 33.5 & 15.9 & 33.8 & 16.3 & 34 & 50.7 & 16.2 & 34.3 & 17.2 & 33.5 \\
\hline $\mathrm{CaO}$ & 21.6 & 0.65 & 22.5 & 0.08 & 0.98 & 22.9 & 0.46 & 22.3 & 0.51 & 0.03 & 21.9 & 0.61 & 21.3 & 0.62 \\
\hline $\mathrm{Na}_{2} \mathrm{O}$ & 1.39 & 0.08 & 0.62 & & 0.05 & 1.44 & 0.05 & 1.34 & 0.06 & & 1.54 & 0.07 & 1.32 & 0.1 \\
\hline $\mathrm{K}_{2} \mathrm{O}$ & 0.00 & 0.00 & 0.01 & 0.00 & 0.00 & 0.00 & 0.00 & 0.00 & 0.00 & 0.00 & 0.00 & 0.00 & 0.01 & 0.00 \\
\hline $\mathrm{P}_{2} \mathrm{O}_{5}$ & 0.01 & 0.01 & 0.01 & 0.02 & 0.01 & 0.01 & 0.01 & 0.01 & 0.01 & 0.01 & 0.01 & 0.01 & 0.02 & 0.01 \\
\hline $\mathrm{Mg} \#$ & 92.6 & 92.1 & 92.2 & 90.9 & 90.9 & 92.8 & 90.8 & 93.3 & 91.4 & 90.8 & 92.8 & 91.6 & 91.9 & 91.2 \\
\hline $\mathrm{Ni}$ & 348 & 734 & 376 & 2809 & 809 & 287 & 691 & 308 & 713 & 3062 & 327 & 715 & 378 & 755 \\
\hline
\end{tabular}




\section{Table DR 2}

Major and trace element, $\mathrm{Sr}-\mathrm{Nd}$ isotopic and C-O isotopic compositions of carbonate components in carbonatite intrusion. Units are wt.\% for major elements, ppm for trace elements and \%o for $\mathrm{C}-\mathrm{O}$ isotopes.

\begin{tabular}{|c|c|c|c|c|c|c|c|c|c|c|c|}
\hline \multicolumn{12}{|c|}{ Carbonatite intrusion (JSB-) } \\
\hline Num. & 12011 & 12022 & 12023 & 12029 & 12032 & 12033 & 12044 & 12046 & 12049 & 12051 & 12053 \\
\hline $\mathrm{SiO}_{2}$ & 5.04 & 3.12 & 3.33 & 5.31 & 2.05 & 4.48 & 4.91 & 4.44 & 0.31 & 1.88 & 6.32 \\
\hline $\mathrm{TiO}_{2}$ & 0.008 & 0.004 & 0.029 & 0.012 & 0.006 & 0.003 & 0.012 & 0.013 & 0.006 & 0.003 & 0.012 \\
\hline $\mathrm{Al}_{2} \mathrm{O}_{3}$ & 0.73 & 0.51 & 0.60 & 0.42 & 0.40 & 0.59 & 1.24 & 1.45 & 0.42 & 0.66 & 1.19 \\
\hline $\mathrm{FeO}$ & 1.41 & 1.03 & 1.57 & 1.63 & 1.32 & 1.55 & 1.11 & 1.30 & 1.17 & 1.04 & 1.28 \\
\hline MgO & 1.39 & 0.90 & 1.04 & 1.58 & 0.88 & 1.08 & 1.35 & 2.18 & 0.80 & 0.85 & 1.59 \\
\hline $\mathrm{CaO}$ & 50.1 & 52.3 & 51.4 & 49.7 & 52.7 & 50.8 & 50.2 & 49.2 & 53.9 & 52.9 & 49.0 \\
\hline $\mathrm{Na}_{2} \mathrm{O}$ & 0.007 & 0.013 & 0.006 & 0.016 & 0.01 & 0.008 & 0.008 & 0.014 & 0.005 & 0.009 & 0.013 \\
\hline $\mathbf{K}_{2} \mathbf{O}$ & 0.019 & 0.014 & 0.02 & 0.023 & 0.014 & 0.017 & 0.014 & 0.016 & 0.012 & 0.026 & 0.016 \\
\hline Sc & 1.02 & 0.40 & 0.97 & 2.25 & 0.89 & 0.80 & 0.69 & 0.65 & 0.36 & 0.29 & 0.80 \\
\hline V & 2.68 & 1.94 & 2.73 & 2.36 & 1.70 & 3.25 & 2.23 & 3.36 & 1.11 & 1.38 & 2.60 \\
\hline $\mathrm{Cr}$ & 61.5 & 35.4 & 56.4 & 104 & 91.5 & 285 & 16.5 & 57.8 & 8.47 & 2.39 & 125 \\
\hline Co & 4.02 & 1.22 & 3.50 & 6.11 & 2.63 & 3.60 & 1.10 & 7.35 & 3.46 & 1.48 & 10.0 \\
\hline $\mathbf{N i}$ & 113 & 58.3 & 118 & 169 & 98.6 & 113 & 74.4 & 181 & 132 & 96.7 & 145 \\
\hline Rb & 0.69 & 0.25 & 0.67 & 0.41 & 0.24 & 0.58 & 0.29 & 0.38 & 0.27 & 0.65 & 0.41 \\
\hline $\mathrm{Sr}$ & 32.4 & 27.5 & 30.6 & 34.2 & 29.8 & 34.3 & 31.7 & 55.7 & 34.1 & 47.0 & 79.1 \\
\hline $\mathbf{Y}$ & 1.72 & 1.20 & 1.29 & 1.68 & 1.16 & 0.87 & 2.30 & 0.53 & 0.56 & 0.55 & 0.50 \\
\hline $\mathrm{Zr}$ & 2.04 & 0.70 & 4.04 & 1.71 & 0.65 & 0.65 & 2.91 & 2.88 & 1.60 & 1.68 & 1.71 \\
\hline Nb & 0.21 & 0.15 & 0.77 & 0.39 & 0.18 & 0.10 & 0.34 & 0.55 & 0.20 & 0.36 & 0.41 \\
\hline Ba & 0.44 & 0.39 & 0.35 & 0.82 & 0.16 & 0.28 & 0.35 & 1.26 & 0.35 & 1.07 & 1.48 \\
\hline La & 1.44 & 1.66 & 1.48 & 1.56 & 1.53 & 1.10 & 2.36 & 0.56 & 0.57 & 0.88 & 0.48 \\
\hline $\mathrm{Ce}$ & 1.34 & 1.73 & 2.24 & 2.16 & 1.91 & 0.83 & 3.54 & 0.67 & 0.58 & 0.69 & 0.63 \\
\hline Pr & 0.37 & 0.27 & 0.31 & 0.39 & 0.27 & 0.15 & 0.62 & 0.11 & 0.11 & 0.12 & 0.095 \\
\hline Nd & 1.73 & 1.16 & 1.43 & 1.79 & 1.12 & 0.69 & 2.85 & 0.49 & 0.46 & 0.48 & 0.43 \\
\hline Sm & 0.43 & 0.22 & 0.35 & 0.42 & 0.23 & 0.14 & 0.73 & 0.099 & 0.10 & 0.10 & 0.089 \\
\hline Eu & 0.25 & 0.12 & 0.13 & 0.20 & 0.12 & 0.089 & 0.32 & 0.049 & 0.056 & 0.042 & 0.034 \\
\hline Gd & 0.41 & 0.23 & 0.28 & 0.40 & 0.26 & 0.15 & 0.60 & 0.11 & 0.10 & 0.098 & 0.079 \\
\hline Tb & 0.07 & 0.03 & 0.04 & 0.06 & 0.03 & 0.02 & 0.08 & 0.01 & 0.01 & 0.01 & 0.01 \\
\hline Dy & 0.36 & 0.14 & 0.22 & 0.30 & 0.14 & 0.11 & 0.43 & 0.081 & 0.080 & 0.059 & 0.078 \\
\hline Ho & 0.07 & 0.03 & 0.04 & 0.05 & 0.03 & 0.02 & 0.08 & 0.01 & 0.02 & 0.01 & 0.02 \\
\hline $\mathbf{E r}$ & 0.17 & 0.063 & 0.094 & 0.12 & 0.071 & 0.050 & 0.19 & 0.036 & 0.040 & 0.029 & 0.034 \\
\hline Tm & 0.028 & 0.008 & 0.011 & 0.019 & 0.009 & 0.006 & 0.024 & 0.005 & 0.005 & 0.004 & 0.004 \\
\hline $\mathbf{Y b}$ & 0.15 & 0.045 & 0.081 & 0.12 & 0.056 & 0.044 & 0.13 & 0.027 & 0.036 & 0.025 & 0.035 \\
\hline Lu & 0.023 & 0.008 & 0.011 & 0.018 & 0.009 & 0.005 & 0.019 & 0.005 & 0.004 & 0.005 & 0.005 \\
\hline Hf & 0.021 & 0.009 & 0.078 & 0.029 & 0.014 & 0.007 & 0.036 & 0.041 & 0.016 & 0.019 & 0.027 \\
\hline Ta & 0.013 & 0.010 & 0.044 & 0.021 & 0.013 & 0.010 & 0.020 & 0.026 & 0.012 & 0.009 & 0.020 \\
\hline $\mathbf{P b}$ & 0.10 & 0.051 & 0.12 & 0.39 & 0.11 & 0.076 & 0.094 & 0.13 & 0.080 & 0.10 & 0.10 \\
\hline Th & 0.02 & 0.01 & 0.06 & 0.03 & 0.01 & 0.01 & 0.02 & 0.02 & 0.01 & 0.03 & 0.02 \\
\hline $\mathbf{U}$ & 0.15 & 0.087 & 0.11 & 0.12 & 0.11 & 0.15 & 0.17 & 0.34 & 0.15 & 0.29 & 0.24 \\
\hline $\mathrm{Ce} / \mathrm{Ce}^{*}$ & 0.45 & 0.63 & 0.80 & 0.68 & 0.72 & 0.50 & 0.71 & 0.66 & 0.56 & 0.51 & 0.71 \\
\hline $\mathbf{E u} / \mathbf{E} \mathbf{u}^{*}$ & 1.78 & 1.62 & 1.17 & 1.50 & 1.42 & 1.80 & 1.44 & 1.40 & 1.62 & 1.27 & 1.21 \\
\hline${ }^{87} \mathrm{Rb} /{ }^{86} \mathrm{Sr}$ & 0.0598 & 0.0256 & 0.0621 & 0.0338 & 0.0223 & 0.0476 & 0.0258 & 0.0192 & 0.0222 & 0.0387 & 0.0145 \\
\hline${ }^{87} \mathrm{Sr} /{ }^{86} \mathrm{Sr}$ & 0.707496 & 0.707576 & 0.707780 & 0.707343 & 0.707710 & 0.707640 & 0.707509 & 0.707963 & 0.707509 & 0.707902 & 0.706383 \\
\hline $2 \sigma$ & 4 & 5 & 5 & 6 & 5 & 7 & 5 & 5 & 17 & 18 & 5 \\
\hline${ }^{147} \mathrm{Sm} /{ }^{144} \mathbf{N d}$ & 0.1564 & 0.1218 & 0.1539 & 0.1479 & 0.1308 & 0.1321 & 0.1600 & 0.1274 & 0.1427 & 0.1306 & 0.1293 \\
\hline${ }^{143} \mathrm{Nd} /{ }^{144} \mathrm{Nd}$ & 0.512869 & 0.512884 & 0.512866 & 0.512887 & 0.51291 & 0.512885 & 0.512909 & 0.512894 & 0.512941 & 0.512872 & 0.512874 \\
\hline $2 \sigma$ & 5 & 4 & 8 & 4 & 4 & 9 & 4 & 7 & 16 & 12 & 13 \\
\hline$\delta^{13} \mathrm{C}_{\text {VPDB }}$ & -12.0 & -12.4 & -12.6 & -13.1 & -11.9 & -12.1 & -11.9 & -11.4 & -12.8 & -11.3 & -12.2 \\
\hline$\delta^{18} O_{\text {SMOW }}$ & 22.7 & 22.8 & 22.6 & 22.6 & 22.8 & 22.8 & 22.7 & 22.7 & 22.5 & 22.6 & 22.3 \\
\hline
\end{tabular}


Table DR 2 continued

Carbonatite intrusion (JSB-)

\begin{tabular}{|c|c|c|c|c|c|c|c|c|c|c|}
\hline Num. & 12055 & 12057 & 12058 & 12061 & 12063 & 12093 & 14005 & 14035 & 14045 & 14009 \\
\hline $\mathrm{SiO}_{2}$ & 7.55 & 2.20 & 0.92 & 5.91 & 2.59 & 3.76 & 7.36 & 3.68 & 16.00 & 2.70 \\
\hline $\mathrm{TiO}_{2}$ & 0.026 & 0.011 & 0.023 & 0.008 & 0.009 & 0.003 & 0.016 & 0.008 & 0.008 & 0.005 \\
\hline $\mathbf{A l}_{2} \mathbf{O}_{3}$ & 1.76 & 0.55 & 1.16 & 0.80 & 0.86 & 0.44 & 1.86 & 0.42 & 0.51 & 1.37 \\
\hline $\mathrm{FeO}$ & 1.27 & 1.13 & 1.64 & 1.13 & 0.91 & 1.17 & 1.28 & 1.45 & 0.52 & 0.73 \\
\hline MgO & 3.00 & 1.18 & 2.03 & 1.10 & 1.43 & 1.24 & 4.81 & 1.56 & 2.03 & 3.35 \\
\hline $\mathrm{CaO}$ & 46.4 & 52.3 & 51.2 & 50.2 & 51.8 & 51.4 & 44.2 & 50.8 & 44.1 & 49.4 \\
\hline $\mathrm{Na}_{2} \mathrm{O}$ & 0.012 & 0.005 & 0.012 & 0.006 & 0.009 & 0.006 & 0.03 & 0.009 & 0.011 & 0.014 \\
\hline $\mathbf{K}_{2} \mathbf{O}$ & 0.015 & 0.013 & 0.02 & 0.013 & 0.024 & 0.014 & 0.012 & 0.012 & 0.039 & 0.013 \\
\hline $\mathrm{Sc}$ & 0.70 & 0.37 & 0.95 & 0.42 & 0.40 & 0.52 & 1.57 & 0.80 & 0.36 & 0.40 \\
\hline $\mathbf{V}$ & 3.10 & 1.63 & 3.51 & 1.25 & 1.58 & 1.47 & 7.08 & 3.41 & 1.70 & 1.70 \\
\hline $\mathrm{Cr}$ & 56.2 & 11.0 & 84.2 & 21.6 & 1.48 & 9.75 & 181 & 112 & 13.6 & 21.7 \\
\hline Co & 5.17 & 4.33 & 6.01 & 2.72 & 1.79 & 1.79 & 8.75 & 3.00 & 1.74 & 1.51 \\
\hline $\mathrm{Ni}$ & 127 & 129 & 198 & 119 & 93.3 & 94.1 & 208 & 152 & 156 & 83.9 \\
\hline $\mathbf{R b}$ & 0.41 & 0.30 & 0.45 & 0.27 & 0.74 & 0.43 & 0.19 & 0.32 & 2.84 & 0.22 \\
\hline $\mathrm{Sr}$ & 109 & 106 & 40.1 & 33.8 & 306 & 68.7 & 64.7 & 72.9 & 138 & 63.3 \\
\hline $\mathbf{Y}$ & 1.82 & 0.69 & 0.94 & 0.62 & 0.62 & 3.26 & 1.83 & 0.86 & 0.45 & 0.72 \\
\hline $\mathbf{Z r}$ & 6.76 & 2.02 & 2.92 & 1.77 & 3.98 & 1.45 & 1.26 & 1.30 & 1.67 & 0.54 \\
\hline $\mathbf{N b}$ & 1.06 & 0.34 & 0.63 & 0.31 & 0.78 & 0.10 & 0.22 & 0.17 & 0.31 & 0.21 \\
\hline $\mathbf{B a}$ & 1.45 & 0.44 & 0.86 & 0.39 & 1.71 & 1.71 & 0.92 & 1.01 & 1.07 & 0.67 \\
\hline La & 2.46 & 0.93 & 1.03 & 0.73 & 0.89 & 2.31 & 1.39 & 0.72 & 0.81 & 1.15 \\
\hline $\mathrm{Ce}$ & 2.82 & 1.09 & 1.58 & 0.96 & 1.07 & 0.55 & 0.45 & 0.56 & 0.57 & 0.95 \\
\hline Pr & 0.41 & 0.15 & 0.23 & 0.14 & 0.15 & 0.31 & 0.23 & 0.10 & 0.11 & 0.15 \\
\hline Nd & 1.73 & 0.67 & 0.97 & 0.67 & 0.65 & 1.38 & 1.06 & 0.46 & 0.40 & 0.58 \\
\hline $\mathrm{Sm}$ & 0.38 & 0.15 & 0.24 & 0.16 & 0.12 & 0.25 & 0.28 & 0.11 & 0.087 & 0.11 \\
\hline $\mathbf{E u}$ & 0.26 & 0.074 & 0.088 & 0.067 & 0.076 & 0.12 & 0.13 & 0.045 & 0.080 & 0.044 \\
\hline Gd & 0.38 & 0.14 & 0.22 & 0.15 & 0.14 & 0.37 & 0.30 & 0.12 & 0.072 & 0.11 \\
\hline $\mathbf{T b}$ & 0.048 & 0.022 & 0.031 & 0.021 & 0.019 & 0.043 & 0.042 & 0.017 & 0.011 & 0.014 \\
\hline Dy & 0.27 & 0.098 & 0.16 & 0.12 & 0.12 & 0.26 & 0.26 & 0.099 & 0.066 & 0.081 \\
\hline Ho & 0.050 & 0.019 & 0.028 & 0.018 & 0.019 & 0.056 & 0.045 & 0.021 & 0.013 & 0.016 \\
\hline $\mathbf{E r}$ & 0.13 & 0.047 & 0.076 & 0.050 & 0.040 & 0.16 & 0.13 & 0.051 & 0.040 & 0.040 \\
\hline $\mathbf{T m}$ & 0.019 & 0.007 & 0.010 & 0.005 & 0.007 & 0.023 & 0.017 & 0.007 & 0.007 & 0.0057 \\
\hline $\mathbf{Y b}$ & 0.12 & 0.045 & 0.055 & 0.040 & 0.038 & 0.14 & 0.11 & 0.044 & 0.041 & 0.030 \\
\hline Lu & 0.016 & 0.005 & 0.010 & 0.006 & 0.006 & 0.023 & 0.018 & 0.007 & 0.008 & 0.006 \\
\hline Hf & 0.073 & 0.033 & 0.053 & 0.029 & 0.037 & 0.009 & 0.027 & 0.017 & 0.024 & 0.010 \\
\hline $\mathbf{T a}$ & 0.046 & 0.018 & 0.038 & 0.014 & 0.019 & 0.008 & 0.013 & 0.010 & 0.024 & 0.028 \\
\hline $\mathbf{P b}$ & 0.12 & 0.082 & 0.16 & 0.066 & 0.22 & 0.11 & 0.063 & 0.088 & 0.22 & 0.082 \\
\hline Th & 0.043 & 0.020 & 0.078 & 0.013 & 0.059 & 0.015 & 0.013 & 0.008 & 0.027 & 0.007 \\
\hline $\mathbf{U}$ & 0.29 & 0.11 & 0.13 & 0.16 & 0.44 & 0.19 & 0.19 & 0.20 & 0.32 & 0.18 \\
\hline $\mathrm{Ce} / \mathrm{Ce}^{*}$ & 0.68 & 0.71 & 0.79 & 0.71 & 0.71 & 0.16 & 0.19 & 0.51 & 0.46 & 0.56 \\
\hline $\mathbf{E u} / \mathbf{E u}{ }^{*}$ & 2.07 & 1.51 & 1.15 & 1.29 & 1.82 & 1.23 & 1.33 & 1.15 & 2.98 & 1.19 \\
\hline${ }^{87} \mathrm{Rb} /{ }^{86} \mathrm{Sr}$ & 0.0105 & 0.0079 & 0.0313 & 0.0224 & 0.0069 & 0.0177 & 0.0082 & 0.0125 & 0.0578 & 0.0098 \\
\hline${ }^{87} \mathrm{Sr} /{ }^{86} \mathrm{Sr}$ & 0.706225 & 0.705401 & 0.707822 & 0.707681 & 0.705222 & 0.707374 & 0.707908 & 0.707108 & 0.706217 & 0.706388 \\
\hline $2 \sigma$ & 6 & 5 & 5 & 6 & 5 & 6 & 5 & 5 & 14 & 10 \\
\hline${ }^{147} \mathrm{Sm} /{ }^{144} \mathrm{Nd}$ & 0.1394 & 0.1413 & 0.1554 & 0.1459 & 0.1141 & 0.1145 & 0.1644 & 0.1562 & 0.1351 & 0.1214 \\
\hline${ }^{143} \mathrm{Nd} /{ }^{144} \mathrm{Nd}$ & 0.512906 & 0.512912 & 0.5129 & 0.512903 & 0.512902 & 0.512871 & 0.512918 & 0.512862 & 0.512817 & 0.512976 \\
\hline $2 \sigma$ & 6 & 4 & 5 & 4 & 6 & 5 & 7 & 14 & 6 & 23 \\
\hline$\delta^{13} C_{\text {VPDB }}$ & -12.7 & -14.4 & -13.5 & -13.3 & -13.6 & -11.3 & -11.9 & -11.8 & -11.4 & -11.2 \\
\hline$\delta^{18} \mathrm{O}_{\text {sMow }}$ & 22.2 & 22.6 & 22.6 & 22.6 & 22.6 & 23.0 & 22.6 & 22.5 & 22.6 & 23.0 \\
\hline
\end{tabular}

Based on the normalization strategy of bulk components as $100 \%, \mathrm{SiO}_{2}{ }^{*}$ is calculated by $100 \%$-the sum of all other major element concentrations expressed as carbonates. 


\section{Table DR 3}

Compositions of calcite phenocryst (CP) and calcite matrix (CM) in the Hannuoba carbonatite intrusion. Units are wt.\% for major elements and ppm for Ni.

\begin{tabular}{|c|c|c|c|c|c|c|c|}
\hline Sample Name & & $\mathrm{CaO}$ & $\mathrm{MgO}$ & $\mathrm{FeO}$ & $\mathrm{SiO}_{2}$ & $\mathrm{Al}_{2} \mathrm{O}_{3}$ & $\mathrm{Ni}$ \\
\hline JSB011-Cc1-01 & $\mathrm{CP}$ & 55.3 & 0.23 & 0.08 & 0.20 & 0.00 & 6.8 \\
\hline JSB011-Cc1-02 & $\mathrm{CP}$ & 55.5 & 0.20 & 0.05 & 0.18 & 0.00 & 1.6 \\
\hline JSB011-Cc1-03 & $\mathrm{CP}$ & 55.4 & 0.22 & 0.04 & 0.26 & 0.00 & 1.4 \\
\hline JSB012-Cc1-01 & $\mathrm{CP}$ & 53.8 & 0.58 & 0.18 & 0.91 & 0.01 & 49.2 \\
\hline JSB012-Cc1-02 & $\mathrm{CP}$ & 54.5 & 0.48 & 0.10 & 0.61 & 0.00 & 34.4 \\
\hline JSB012-Cc1-03 & $\mathrm{CP}$ & 54.4 & 0.61 & 0.08 & 0.50 & 0.00 & 39.4 \\
\hline JSB012-Cc2-01 & $\mathrm{CP}$ & 54.7 & 0.60 & 0.03 & 0.44 & 0.00 & 2.7 \\
\hline JSB012-Cc2-02 & $\mathrm{CP}$ & 54.6 & 0.68 & 0.03 & 0.40 & 0.00 & 3.2 \\
\hline JSB012-Cc2-03 & $\mathrm{CP}$ & 54.8 & 0.59 & 0.03 & 0.34 & 0.00 & 3.3 \\
\hline JSB024-Cc1-01 & $\mathrm{CP}$ & 53.7 & 1.42 & 0.06 & 0.36 & 0.00 & 3.9 \\
\hline JSB024-Cc1-02 & $\mathrm{CP}$ & 53.5 & 1.62 & 0.06 & 0.41 & 0.00 & 3.4 \\
\hline JSB024-Cc1-03 & $\mathrm{CP}$ & 53.9 & 1.33 & 0.08 & 0.28 & 0.00 & 1.5 \\
\hline JSB024-Cc2-01 & $\mathrm{CP}$ & 54.5 & 0.63 & 0.07 & 0.49 & 0.01 & 9.3 \\
\hline JSB024-Cc2-02 & $\mathrm{CP}$ & 54.5 & 0.45 & 0.13 & 0.58 & 0.02 & 13.2 \\
\hline JSB024-Cc2-03 & $\mathrm{CP}$ & 54.9 & 0.36 & 0.07 & 0.42 & 0.01 & 8.0 \\
\hline JSB029-Cc1-01 & $\mathrm{CP}$ & 53.6 & 1.63 & 0.08 & 0.28 & 0.00 & 1.5 \\
\hline JSB029-Cc1-02 & $\mathrm{CP}$ & 53.6 & 1.59 & 0.09 & 0.31 & 0.00 & 1.8 \\
\hline JSB029-Cc1-03 & $\mathrm{CP}$ & 53.2 & 1.65 & 0.12 & 0.50 & 0.02 & 10.3 \\
\hline JSB029-Cc2-01 & $\mathrm{CP}$ & 54.2 & 0.58 & 0.12 & 0.64 & 0.03 & 11.7 \\
\hline JSB029-Cc2-02 & $\mathrm{CP}$ & 48.9 & 1.04 & 0.98 & 3.30 & 0.21 & 98.9 \\
\hline JSB044-Cc1-04 & $\mathrm{CP}$ & 53.7 & 1.61 & 0.02 & 0.30 & 0.00 & 5.6 \\
\hline JSB044-Cc1-05 & $\mathrm{CP}$ & 53.9 & 1.54 & 0.02 & 0.21 & 0.00 & 4.9 \\
\hline JSB044-Cc1-06 & $\mathrm{CP}$ & 53.6 & 1.74 & 0.02 & 0.23 & 0.00 & 4.9 \\
\hline JSB044-Cc2-03 & $\mathrm{CP}$ & 54.8 & 0.26 & 0.09 & 0.53 & 0.04 & 8.0 \\
\hline JSB044-Cc2-04 & $\mathrm{CP}$ & 54.9 & 0.39 & 0.08 & 0.40 & 0.03 & 7.9 \\
\hline JSB044-Cc2-05 & $\mathrm{CP}$ & 51.6 & 0.46 & 0.50 & 1.99 & 0.40 & 41.8 \\
\hline JSB044-Cc2-06 & $\mathrm{CP}$ & 54.2 & 0.26 & 0.20 & 0.81 & 0.13 & 15.0 \\
\hline JSB054-Cc1-01 & $\mathrm{CP}$ & 54.7 & 0.30 & 0.06 & 0.48 & 0.08 & 7.9 \\
\hline JSB054-Cc1-02 & $\mathrm{CP}$ & 54.9 & 0.47 & 0.03 & 0.33 & 0.01 & 4.4 \\
\hline JSB054-Cc3-05 & $\mathrm{CP}$ & 54.0 & 0.43 & 0.18 & 0.84 & 0.05 & 19.7 \\
\hline JSB054-Cc3-06 & $\mathrm{CP}$ & 54.4 & 0.53 & 0.12 & 0.55 & 0.03 & 17.0 \\
\hline JSB054-Cc3-07 & $\mathrm{CP}$ & 52.8 & 0.44 & 0.42 & 1.39 & 0.17 & 37.9 \\
\hline JSB054-Cc3-08 & $\mathrm{CP}$ & 53.6 & 0.31 & 0.24 & 1.07 & 0.16 & 26.9 \\
\hline JSB054-Cc3-09 & $\mathrm{CP}$ & 55.2 & 0.31 & 0.03 & 0.30 & 0.00 & 4.4 \\
\hline JSB054-Cc3-10 & $\mathrm{CP}$ & 54.4 & 0.39 & 0.16 & 0.70 & 0.04 & 18.0 \\
\hline JSB057-Cc3-02 & $\mathrm{CP}$ & 55.2 & 0.19 & 0.07 & 0.32 & 0.00 & 5.1 \\
\hline JSB057-Cc3-03 & $\mathrm{CP}$ & 55.3 & 0.20 & 0.06 & 0.29 & 0.00 & 5.4 \\
\hline JSB057-Cc1-01 & $\mathrm{CP}$ & 52.0 & 0.53 & 0.53 & 1.79 & 0.16 & 65.8 \\
\hline JSB057-Cc1-02 & $\mathrm{CP}$ & 52.6 & 0.40 & 0.43 & 1.59 & 0.19 & 55.7 \\
\hline JSB057-Cc1-03 & $\mathrm{CP}$ & 53.7 & 0.32 & 0.31 & 1.05 & 0.07 & 38.1 \\
\hline JSB057-Cc1-04 & $\mathrm{CP}$ & 50.8 & 0.45 & 0.72 & 2.54 & 0.34 & 88.6 \\
\hline
\end{tabular}


Table DR 3 continued

\begin{tabular}{clllllll}
\hline Sample Name & & $\mathrm{CaO}$ & $\mathrm{MgO}$ & $\mathrm{FeO}$ & $\mathrm{SiO}_{2}$ & $\mathrm{Al}_{2} \mathrm{O}_{3}$ & $\mathrm{Ni}$ \\
\hline JSB011-Cc2-01 & $\mathrm{CM}$ & 51.2 & 0.48 & 0.94 & 2.31 & 0.11 & 36.8 \\
JSB011-Cc2-02 & $\mathrm{CM}$ & 51.1 & 0.48 & 0.98 & 2.27 & 0.14 & 36.1 \\
JSB011-Cc2-03 & $\mathrm{CM}$ & 52.7 & 0.41 & 0.58 & 1.54 & 0.08 & 21.7 \\
JSB011-Cc2-04 & $\mathrm{CM}$ & 52.1 & 0.48 & 0.74 & 1.78 & 0.08 & 24.7 \\
JSB024-Cc3-01 & $\mathrm{CM}$ & 51.6 & 0.54 & 0.79 & 2.06 & 0.05 & 36.0 \\
JSB024-Cc3-02 & $\mathrm{CM}$ & 49.8 & 0.69 & 1.15 & 2.97 & 0.08 & 62.9 \\
JSB024-Cc3-03 & $\mathrm{CM}$ & 50.3 & 0.69 & 1.06 & 2.74 & 0.06 & 57.5 \\
JSB024-Cc3-04 & $\mathrm{CM}$ & 48.8 & 0.72 & 1.27 & 3.53 & 0.14 & 104 \\
JSB024-Cc3-05 & $\mathrm{CM}$ & 49.8 & 0.62 & 1.07 & 3.10 & 0.11 & 101 \\
JSB029-Cc1-01 & $\mathrm{CM}$ & 48.0 & 1.03 & 1.57 & 3.74 & 0.08 & 55.9 \\
JSB029-Cc1-02 & $\mathrm{CM}$ & 46.8 & 1.20 & 1.85 & 4.30 & 0.06 & 54.2 \\
JSB044-Cc1-01 & $\mathrm{CM}$ & 51.0 & 0.47 & 0.88 & 2.37 & 0.23 & 29.1 \\
JSB044-Cc1-02 & $\mathrm{CM}$ & 47.1 & 0.90 & 1.49 & 4.12 & 0.49 & 68.3 \\
JSB044-Cc1-03 & $\mathrm{CM}$ & 46.2 & 0.91 & 1.81 & 4.63 & 0.43 & 133 \\
JSB044-Cc1-07 & $\mathrm{CM}$ & 51.5 & 0.53 & 0.82 & 2.00 & 0.18 & 44.7 \\
JSB044-Cc1-08 & $\mathrm{CM}$ & 51.6 & 0.51 & 0.79 & 2.03 & 0.16 & 38.4 \\
JSB044-Cc2-01 & $\mathrm{CM}$ & 51.1 & 0.60 & 0.85 & 2.24 & 0.22 & 34.7 \\
JSB044-Cc2-02 & $\mathrm{CM}$ & 48.4 & 0.66 & 1.56 & 3.64 & 0.23 & 56.2 \\
JSB054-Cc3-01 & $\mathrm{CM}$ & 47.5 & 0.74 & 1.07 & 4.00 & 0.82 & 80.5 \\
JSB054-Cc3-02 & $\mathrm{CM}$ & 49.8 & 0.63 & 0.79 & 2.91 & 0.50 & 62.9 \\
JSB054-Cc3-03 & $\mathrm{CM}$ & 49.7 & 0.60 & 0.77 & 2.99 & 0.50 & 63.2 \\
JSB054-Cc3-04 & $\mathrm{CM}$ & 51.1 & 0.52 & 0.74 & 2.33 & 0.26 & 50.0 \\
JSB057-Cc3-01 & $\mathrm{CM}$ & 52.5 & 0.47 & 0.60 & 1.65 & 0.02 & 35.8 \\
JSB057-Cc3-04 & $\mathrm{CM}$ & 50.8 & 0.62 & 0.93 & 2.48 & 0.04 & 80.5 \\
JSB054-Cc1-03 & $\mathrm{CM}$ & 49.6 & 1.11 & 0.69 & 2.89 & 0.31 & 76.5 \\
JSB054-Cc1-04 & $\mathrm{CM}$ & 45.2 & 1.80 & 0.87 & 4.97 & 0.70 & 116 \\
\hline
\end{tabular}




\section{Table DR 4}

$\mathrm{Sr}$ content and $\mathrm{Sr}$ isotopic compositions of aragonite veinlets crosscutting one sample of the carbonatite intrusion.

\begin{tabular}{cccc}
\hline Sample & $\mathbf{S r}(\mathbf{p p m})$ & ${ }^{\mathbf{8 7}} \mathbf{S r}{ }^{\mathbf{8 6}} \mathbf{S r}$ & $\mathbf{2 \sigma}$ \\
\hline JSB15062-1 & 6022 & 0.70605 & 0.000015 \\
JSB15062-2 & 1610 & 0.70571 & 0.000039 \\
JSB15062-3 & 2700 & 0.70635 & 0.000034 \\
JSB15062-4 & 4909 & 0.70673 & 0.000016 \\
JSB15062-5 & 7848 & 0.70672 & 0.000013 \\
JSB15062-6 & 3654 & 0.70560 & 0.000019 \\
JSB15062-7 & 1545 & 0.70532 & 0.000047 \\
JSB15062-8 & 6442 & 0.70583 & 0.000027 \\
JSB15062-9 & 5005 & 0.70683 & 0.000017 \\
JSB15062-10 & 5005 & 0.70684 & 0.000021 \\
JSB15062-11 & 4941 & 0.70584 & 0.000030 \\
JSB15062-13 & 3246 & 0.70411 & 0.000039 \\
\hline
\end{tabular}




\section{References}

Armstrong-Altrin, J. S., Verma, S. P., Madhavaraju, J., Lee, Y. I., and Ramasamy, S., 2003, Geochemistry of Upper Miocene Kudankulam Limestones, Southern India: International Geology Review, v. 45, p. 16-26, doi: 10.2747/0020-6814.45.1.16.

Bühn, B., 2008, The role of the volatile phase for REE and Y fractionation in low-silica carbonate magmas: implications from natural carbonatites, Namibia: Mineralogy and Petrology, v. 92, p. 453-470, doi: 10.1007/s00710-007-0214-4.

Bell, K., Blenkinsop, J., Cole, T. J. S., and Menagh, D. P., 1982, Evidence from Sr isotopes for long-lived heterogeneities in the upper mantle: Nature, v. 298, p. 251-253, doi: $10.1038 / 298251 \mathrm{a} 0$.

Bell, K., and Simonetti, A., 2010, Source of parental melts to carbonatites-critical isotopic constraints: Mineralogy and Petrology, v. 98, p. 77-89, doi: 10.1007/s00710-009-0059-0.

Bellanca, A., Masetti, D., and Neri, R., 1997, Rare earth elements in limestone/marlstone couplets from the Albian-Cenomanian Cismon section (Venetian region, northern Italy) : assessing REE sensitivity to environmental changes: Chemical Geology, v. 141, p. 141-152, doi: 10.1016/S0009-2541(97)00058-2.

Burke, K., Ashwal, L. D., and Webb, S. J., 2003, New way to map old sutures using deformed alkaline rocks and carbonatites: Geology, v. 31, p. 391-394.

Chen, L., Liu, Y. S., Hu, Z. C., Gao, S., Zong, K. Q., and Chen, H. H., 2011, Accurate determinations of fifty-four major and trace elements in carbonate by LA-ICP-MS using normalization strategy of bulk components as 100\%: Chemical Geology, v. 284, p. 283-295, doi: 10.1016/j.chemgeo.2011.03.007.

Gao, S., et al., 2004, Recycling lower continental crust in the North China craton: Nature, v. 432, p. 892-897, doi: 10.1038/nature03162.

Halama, R., McDonough, W. F., Rudnick, R. L., and Bell, K., 2008, Tracking the lithium isotopic evolution of the mantle using carbonatites: Earth and Planetary Science Letters, v. 265, p. 726-742, doi: 10.1016/j.epsl.2007.11.007.

Hoernle, K., Tilton, G., Le Bas, M., Duggen, S., and Garbe-Schönberg, D., 2002, Geochemistry of oceanic carbonatites compared with continental carbonatites: mantle recycling of oceanic crustal carbonate: Contributions to Mineralogy and Petrology, v. 142, p. 520-542, doi: 10.1007/s004100100308.

Hou, Z. Q., Liu, Y., Tian, S. H., Yang, Z. M., and Xie, Y. L., 2015, Formation of carbonatite-related giant rare-earth-element deposits by the recycling of marine sediments: Sci Rep, v. 5, p. 10231, doi: $10.1038 /$ srep10231.

Huang, Y. M., Hawkesworth, C. J., van Calsteren, P., and McDermott, F., 1995, Geochemical characteristics and origin of the Jacupiranga carbonatites, Brazil: Chemical Geology, v. 119, p. 79-99, doi: 10.1016/0009-2541(94)00093-N.

Jin, Z. J., Zhu, D. Y., Hu, W. X., Zhang, X. F., Zhang, J. T., and Song, Y. C., 2009, Mesogenetic dissolution of the middle Ordovician limestone in the Tahe oilfield of Tarim basin, NW China: Marine and Petroleum Geology, v. 26, p. 753-763, doi: 10.1016/j.marpetgeo.2008.08.005.

Jolis, E. M., Freda, C., Troll, V. R., Deegan, F. M., Blythe, L. S., McLeod, C. L., and Davidson, J. P., 2013, Experimental simulation of magma-carbonate interaction beneath Mt. Vesuvius, Italy: 
Contributions to Mineralogy and Petrology, v. 166, p. 1335-1353, doi: 10.1007/s00410-013-0931-0.

Keto, L. S., and Jacobsen, S. B., 1988, Nd isotopic variations of Phanerozoic paleoceans: Earth and Planetary Science Letters, v. 90, p. 395-410, doi: 10.1016/0012-821X(88)90138-0.

Klein, C., and Beukes, N. J., 1989, Geochemistry and sedimentology of a facies transition from limestone to iron-formation deposition in the early Proterozoic Transvaal Supergroup, South Africa.: Economic Geology, v. 84, p. 1733-1774.

Liu, Y. S., Gao, S., Lee, C.-T. A., Hu, S. H., Liu, X. M., and Yuan, H. L., 2005, Melt-peridotite interactions: Links between garnet pyroxenite and high-Mg\# signature of continental crust: Earth and Planetary Science Letters, v. 234, p. 39-57, doi: 10.1016/j.eps1.2005.02.034.

Liu, Y. S., He, D. T., Gao, C. G., Foley, S. F., Gao, S., Hu, Z. C., Zong, K. Q., and Chen, H. H., 2015, First direct evidence of sedimentary carbonate recycling in subduction-related xenoliths: Scientific Reports, v. 5, p. 11547, doi: 10.1038/srep11547.

Liu, Y. S., Zong, K. Q., Kelemen, P. B., and Gao, S., 2008a, Geochemistry and magmatic history of eclogites and ultramafic rocks from the Chinese continental scientific drill hole: Subduction and ultrahigh-pressure metamorphism of lower crustal cumulates: Chemical Geology, v. 247, p. 133-153, doi: 10.1016/j.chemgeo.2007.10.016.

Liu, Y. S., Hu, Z. C., Gao, S., Guenther, D., Xu, J., Gao, C. G., and Chen, H. H., 2008b, In situ analysis of major and trace elements of anhydrous minerals by LA-ICP-MS without applying an internal standard: Chemical Geology, v. 257, p. 34-43, doi: 10.1016/j.chemgeo.2008.08.004.

Mitchell, R. H., 2005, Carbonatites and carbonatites and carbonatites.: The Canadian Mineralogist, v. 43, p. 2049-2068, doi: 10.2113/gscanmin.43.6.2049.

Mourao, C., Mata, J., Doucelance, R., Madeira, J., da Silveira, A. B., Silva, L. C., and Moreira, M., 2010, Quaternary extrusive calciocarbonatite volcanism on Brava Island (Cape Verde): A nephelinite-carbonatite immiscibility product: Journal of African Earth Sciences, v. 56, p. 59-74, doi: 10.1016/j.jafrearsci.2009.06.003.

Rudnick, R. L., Gao, S., Ling, W. L., Liu, Y. S., and McDonough, W. F., 2004, Petrology and geochemistry of spinel peridotite xenoliths from Hannuoba and Qixia, North China craton: Lithos, v. 77, p. 609-637, doi: 10.1016/j.lithos.2004.03.033.

Song, Y., Frey, F. A., and Zhi, X., 1990, Isotopic characteristics of Hannuoba basalts, eastern China: Implications for their petrogenesis and the composition of subcontinental mantle: Chemical Geology, v. 88, p. 35-52, doi: 10.1016/0009-2541(90)90102-D.

Tanaka, K., Miura, N., Asahara, Y., and Kawabe, I., 2003, Rare earth element and strontium isotopic study of seamount-type limestones in Mesozoic accretionary complex of Southern Chichibu Terrane, central Japan: Implication for incorporation process of seawater REE into limestones: Geochemical Journal, v. 37, p. 163-180.

Tsikos, H., Moore, J. M., and Harris, C., 2001, Geochemistry of the Palæoproterozoic Mooidraai Formation: Fe-rich limestone as end member of iron formation deposition, Kalahari Manganese Field, Transvaal Supergroup, South Africa: Journal of African Earth Sciences, v. 32, p. 19-27, doi: 10.1016/S0899-5362(01)90016-8.

Veizer, J., 1985, Carbonates and Ancient Oceans: Isotopic and Chemical Record on Time Scales of 107-109 Years, The Carbon Cycle and Atmospheric CO2: Natural Variations Archean to Present, American Geophysical Union, p. 595-601.

Veizer, J., et al., 1999, ${ }^{87} \mathrm{Sr} /{ }^{86} \mathrm{Sr}, \delta^{13} \mathrm{C}$ and $\delta^{18} \mathrm{O}$ evolution of Phanerozoic seawater: Chemical Geology, v. 
161, p. 59-88, doi: 10.1016/S0009-2541(99)00081-9.

Wells, P. R. A., 1977, Pyroxene thermometry in simple and complex systems: Contributions to Mineralogy and Petrology, v. 62, p. 129-139, doi: 10.1007/bf00372872.

Xu, R., Liu, Y. S., Tong, X. R., Hu, Z. C., Zong, K. Q., and Gao, S., 2013, In-situ trace elements and Li and $\mathrm{Sr}$ isotopes in peridotite xenoliths from Kuandian, North China Craton: Insights into Pacific slab subduction-related mantle modification: Chemical Geology, v. 354, p. 107-123, doi: 10.1016/j.chemgeo.2013.06.022.

$\mathrm{Xu}$, Y. G., 2002, Evidence for crustal components in the mantle and constraints on crustal recycling mechanisms: pyroxenite xenoliths from Hannuoba, North China: Chemical Geology, v. 182, p. 301-322, doi: 10.1016/S0009-2541(01)00300-X.

Yan, G. H., Mu, B. L., Zeng, Y. S., Cai, J. H., Ren, K. X., and Li, F. T., 2007, Igneous Carbonatites in North China Craton: The Temporal and Spatial Distribution, Sr and Nd Isotopic Charateristics and Their Geological Significance (in Chinese with English abstract): Geological Journal of China Universities, v. 13, p. 463-473. 